\title{
Survey on Satellite Image Extraction using Texture and Colour Feature
}

\author{
Anjali M. Damani ${ }^{1} \&$ Rushabh D. Doshi ${ }^{2}$ \\ ${ }^{1}$ M.E Student, Department of Computer Engineering, V.V.P Engineering College, Rajkot, Gujarat, India \\ ${ }^{2}$ Lecturer, V.V.P Engineering College, Rajkot, Gujarat, India
}

\begin{abstract}
Satellite image processing is a significant field in research and development. Many image processing techniques have been developed to extract as much information as possible from the image. There are many parameters for retrieving information from images like colour, shape, texture, edge etc. In this paper, parameters texture and colour are used for extracting information from image. Technique for texture extraction used here is, GLCM . The detailed analysis of result of image retrieved using SURF descriptor ,CS SURF descriptor is also given.
\end{abstract}

Keywords - Satellite, image classification, texture, colour, SURF.

\section{INTRODUCTION}

The most common and convenient means of transmitting information are Images. Satellite images are very consequential and sturdy tools for meteorological and scientist. satellite images give a good representation of what is happening at every point in the world. The three most common types of satellite images are:(1)Visible satellite image(2)Infrared (IR) satellite image(3)water vapour satellite image. Image processing is a method to extract some meaningful information from it. A type of signal processing in which input is an image and output may be image or characteristics/features associated with that image is also known as Image processing. Satellite images coincide features like colour, texture, shape, brightness, radiated energy frequency etc.It is most important to retrieve as best image as possible by satellite, after successful launching of the satellite. Single feature extraction can not give as effective result as multiple feature extraction can give. In this Paper, we are surveying 4 paper for information retrieval from satellite image. For texture extraction various techniques available are , gabor, GLCM, kohnon self organizing etc, among them GLCM gives quality result. So For effective extracting of texture feature GLCM is used here and SURF is used for standard colour extraction.

\section{CBIR SYSTEM WITH COLOUR AND TEXTURE[2]}

As shown in figure-1,Basically CBIR uses two perspective for retrieving the images from the image data base. One is Textbased perspective (index images using keywords), that use the keywords descriptions as a input and then the desired output is obtained in the form of similar types of images. Content-Based perspective is another approach, which uses image as an input query and it generate the output of similar types of images. An image consists of complex structure and also having various levels of details. Single resolution is not sufficient to determine the details present within an image. Multiple resolution analysis overcomes this drawback. Multiple resolution analysis analyses images at more than one resolution so that the features that are left undetected at one level get spotted at another level. This paper include the combination of colour and texture features at more than one resolution of image in order to combine advantages of multiple features to form efficient and powerful feature vector for image retrieval, In this paper, colour information is extracted by using SURF descriptor and texture is extracted by using method GLCM and gabor filter.

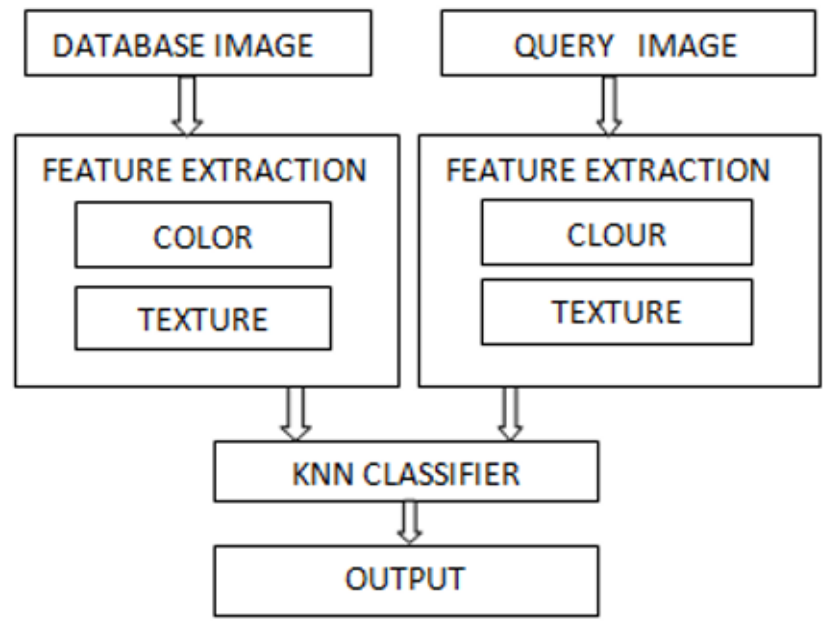

Fig -1 CBIR system 
In given figure-1, KNN classifier is used to classify the images which use Euclidean distance to find out similarity between features.

\section{INFORMATION FUSION CBIR IN SYSTEM[3]}

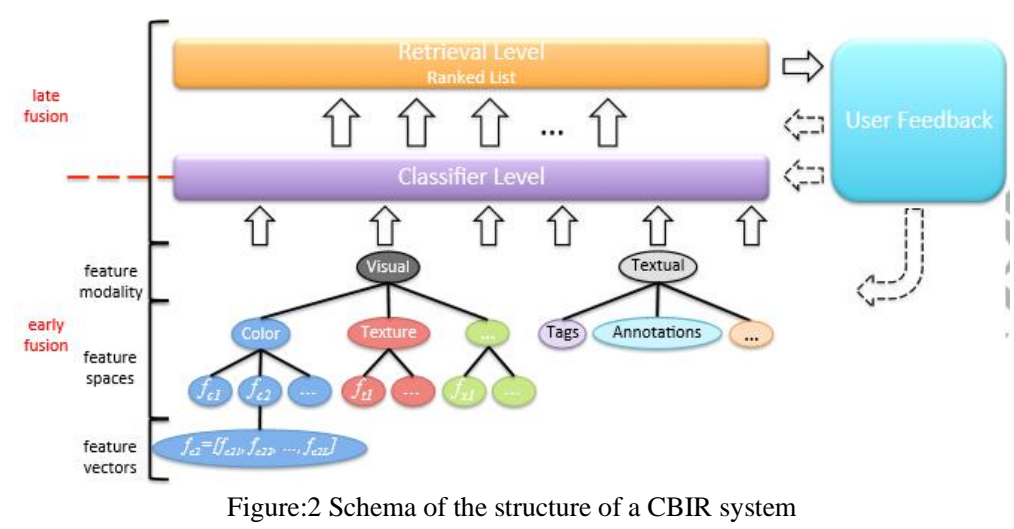

As shown in figure:2,Information fusion in CBIR has two classes. First is early fusion and second is late fusion. early fusion usually allod to the combination of the features into a single representation before the computation of similarity between images. This approach is well known and simple. based on the concatenation of the feature vectors into a single vector. While Late fusion refers either to the combination of the outputs produced by different retrieval systems, or to the combination of the similarity rankings, the outputs and the rankings referring to different feature representations.

\section{(4) TEXTURE EXTRACTION USING GLCM APPROACH: [5]}

Texture is one of the significant characteristics used in identifying objects or in detecting regions of interest in an image. Texture contains crucial information about the structural arrangement of surfaces. Textural features contain information about the spatial distribution. The GLCM contain information about image texture characteristics such as homogeneity, gray-tone linear dependencies, contrast, number and nature of boundaries present and the complexity of the image. In texture based approach, contextual features contain information derived from blocks of pictorial data surrounding the area being analysed. GLCM set of features are based on second order statistics. They can be used to reflect, the overall average for degree of correlation between pairs of pixels in different aspects like in terms of homogeneity, uniformity etc. Few of the common statistics applied to co-occurrence probabilities are discussed below.

\section{(4.1) Angular Second Moment}

This statistic is also called Uniformity or Energy. It is used to measure the textural uniformity that is pixel pair repetitions. Energy detects disorders in texture. It is the sum of squares of entries in the GLCM angular second moment and measures the image homogeneity. High energy values occurs when image has good homogeneity or when pixels are very similar. It means, angular second moment is high when gray level distribution has a constant or periodic form.

Energy: $\Sigma \Sigma p 2(i, j)$

Where $i, j$ are the spatial coordinates of the function $p(i, j)$.

(4.2) Entropy:

This statistic measures the disorder or complexity of an image. It is large when the image is not texturally uniform and many GLCM elements will have very small values. Complex textures tend to have high entropy. It is strongly, but inversely correlated to energy.

Entropy $=\sum \sum(\mathrm{i}, \mathrm{j})_{(2)} \log [\mathrm{P}(\mathrm{i}, \mathrm{j})]$

(4.3) Variance:

Heterogeneity is measured by it and variance is strongly correlated with standard deviation. When the gray level values differ from their mean, variance increases.

Variance $=\sum \sum(\mathrm{i}-\mu)^{2}$ gij $\quad$ where $\mu$ is the mean of $g_{i j}$

(4.4) Homogeneity:

This statistic is also called as Inverse Difference Moment. It is used to measure an image homogeneity as it assumes larger values for smaller gray tone differences in pair elements. Homogeneity is more sensitive to the presence of near diagonal elements in the GLCM. Homogeneity has maximum value when all elements in the image are same. Contrast and homogeneity of GLCM are strongly, but inversely, correlated in terms of equivalent distribution in the pixel pairs population. If energy is kept constant, homogeneity decreases if contrast increases.

Homogeneity $=\sum \sum \frac{1}{1+(i-j) 2}$ gij

(4.5) Correlation:

Correlation $=\frac{\sum \sum(\mathrm{ij}) \mathrm{gij}-\mu \mathrm{x} \mu \mathrm{y}}{\sigma x \sigma y}$ 
where $\mu \mathrm{x}, \mu \mathrm{y}, \sigma \mathrm{x}$ and $\sigma \mathrm{y}$ are the means and standard deviations of gx and gy It is a measure of gray tone linear dependencies in the image.

\section{(4.6) Mean:}

Mean $=\sum \sum \mathrm{i}(\mathrm{i})$

It represents amount of brightness present in an image.[2]

(4.7) Process used to create the GLCM

\begin{tabular}{|c|c|c|c|c|c|c|c|c|c|c|c|c|c|}
\hline 1 & 1 & 5 & 6 & 8 & & 1 & 2 & 3 & 4 & 5 & 6 & 7 & 8 \\
\hline & & & & & 1 & 1 & 2 & 0 & 0 & 1 & 0 & 0 & 0 \\
\hline$?$ & 3 & 15 & 7 & 1 & 2 & 0 & B & 1 & 0 & 0 & 0 & 0 & 1 \\
\hline 2 & 3 & J & t & 1 & 3 & 0 & 0 & 0 & 0 & 1 & 0 & 0 & 0 \\
\hline & & & & & 4 & 0 & 0 & 0 & 0 & 1 & 0 & 0 & 0 \\
\hline 4 & 5 & 7 & 1 & 2 & 5 & 0 & 1 & 0 & 0 & 1 & 1 & 2 & 0 \\
\hline & & & & & 6 & 0 & 0 & 0 & 0 & 0 & 0 & 0 & 1 \\
\hline 1 & 2 & 8 & 5 & 5 & 7 & 2 & 0 & 0 & 0 & 0 & 0 & 0 & 0 \\
\hline & & & & & 8 & 0 & 0 & 0 & 0 & 1 & 0 & 0 & 0 \\
\hline
\end{tabular}

Table:1classical co-occurrence Matrix

The above Table shows how graycomatrix calculates the first three values in GLCM. In the output GLCM, $(1,1)$ contains value 1 because there is only one instance in the input image where two horizontally adjacent pixels have the value 1 and 1 , respectively. graycomatrix continues processing the input image, scanning the image for other pixel pairs(i,j) and recording the sums in the corresponding elements of the GLCM. Now,we will see SURF descriptor for colour extraction.

\section{(5) SURF: FEATURE DETECTION \& DESSCRIPTION[8]}

Speeded Up Robust Features is patented algorithm used mostly in computer vision tasks and tied to object detection purpose. SURF is partially simulated by SIFT(Scale Invariant Feature Transformation)descriptor. Basic Version of SURF is three time faster than SIFT.

\section{Main stages of SURF algorithm[6]}

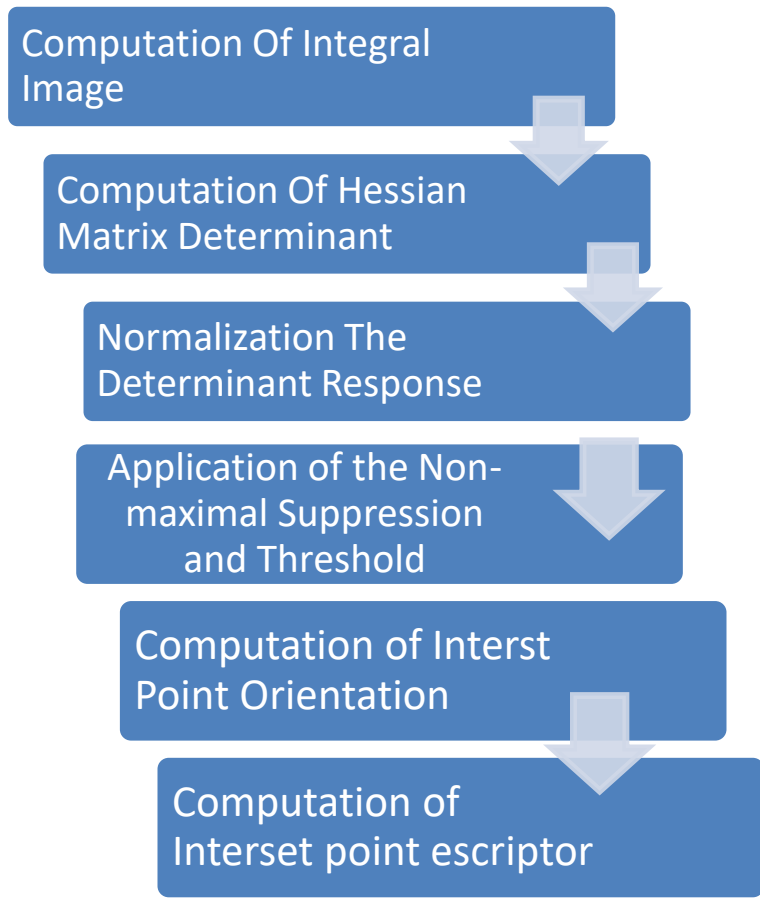

$\mathrm{S}(\mathrm{x}, \mathrm{y})=\sum_{i=0}^{x} \sum_{j=0}^{y} I(i, j)$

Figure:3 Main Stages of SURF algorithm

In SURF algorithm the sum of the original image within a rectangle can be evaluated quickly using the integral image, requiring evaluations at the rectangle's four corners. Then SURF use blob detector based on Hessian matrix to find points of interest. This is used as a measure of local change around the point and points are chosen where this determinant is maximal. 
The Hessian matrix $\mathrm{H}(\mathrm{p}, \sigma)$ At given point $\mathrm{p}$ and scale $\sigma$,is

$\mathrm{H}(\mathrm{p}, \sigma)=\left(\begin{array}{ll}\operatorname{Lxx}(p, \sigma) & \operatorname{Lxy}(p, \sigma) \\ \operatorname{Lxx}(p, \sigma) & \operatorname{Lxy}(p, \sigma)\end{array}\right)$

Where $\operatorname{Lxx}(p, \sigma)$ etc.is convolution of second-order derivative of gussian with the image I(x,y) at the point $\mathrm{p}$.[6]In other feature detection algorithms, the scale space is usually realized as an image pyramid. images are repeatedly smoothed with a Gaussian filter, then they are subsampled to get the next higher level or stairs with various measured the masks are calculated. The SURF descriptor is extracted from an image by firstly assigning an orientation to each interest point. The orientation is computed using Haar-wavelet responses in both $\mathrm{x}$ and $\mathrm{y}$ direction. In a second step, the region around the interest point is split up regularly into smaller square sub-regions and a few simple features at regularly spaced sample points are computed for each sub-region. The horizontal and vertical wavelet responses $\mathrm{dx}$ and dy are summed up over each sub-region. Furthermore, the absolute values I dx I and I dy I are summed in order to obtain information about the polarity of the image intensity changes. Therefore, each subregion has a four-dimensional descriptor Vector:The resulting descriptor vector for all 4 by 4 sub-regions is of length.
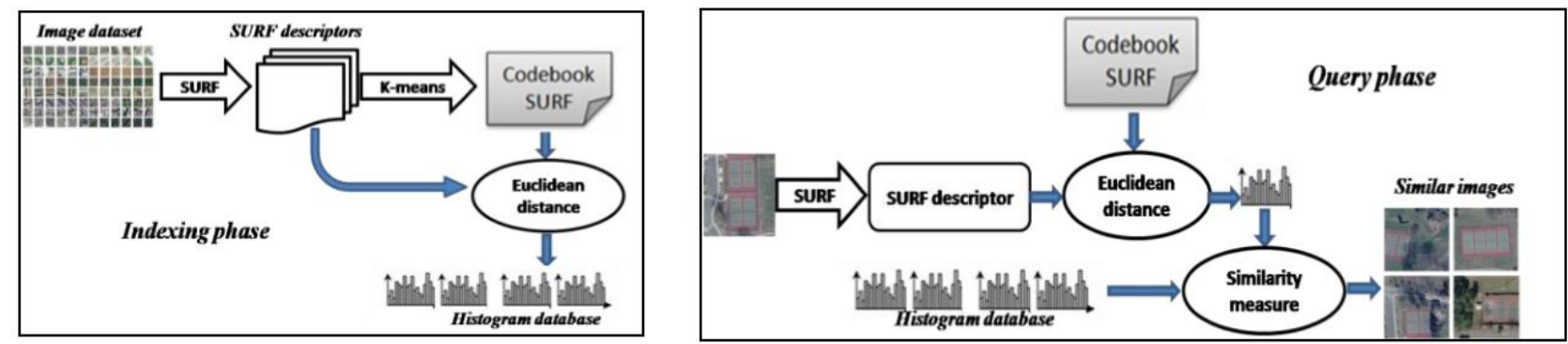

Figure-4 Indexing and Query phase

As shown in figure-4,For effective control the influence of the colour information to the SURF description, here we added weights to the distances of each before constructing the histograms: Distance $=\mathrm{k} 1 * \mathrm{SURF}$ distance $+\mathrm{k} 2 *$ colour distance. The second alternative is to create two codebooks, one for the SURF descriptor and another one for the colour descriptor. Each image is therefore Depicted by two histograms. Similarly, the coefficients are added to weight each description. The third alternative is to generate a SURF codebook for each colour band.

Table 2,given below illustrates the obtained results in terms of different percentages of relevant images over the retrieved images. It shows that, using the category-specific(CS) learned dictionary, the overall performance is improved by $3 \%$ for SURF and more than $1.5 \%$ for SURF combined to colour[1]. In addition to the global image dataset and for comparion purposes, we conducted a second test on a reduced set, consisting of 8 classes that are: agricultural,airplane,beach,buildings,chaparral,denseresidential, forest and harbour.

\begin{tabular}{|c|c|c|c|c|c|c|c|c|c|c|c|}
\hline Retrieved images & $10 \%$ & $20 \%$ & $30 \%$ & $40 \%$ & $50 \%$ & $60 \%$ & $70 \%$ & $80 \%$ & $90 \%$ & $100 \%$ & Average \\
\hline SURF & 68,68 & 62,21 & 58,49 & 55,74 & 53,38 & 51,21 & 49,17 & 47,17 & 45,27 & 43,31 & 53,46 \\
\hline CS SURF & 70,67 & 64,57 & 61,07 & 58,55 & 56,45 & 54,46 & 52,60 & 50,75 & 48,79 & 46,72 & 56,46 \\
\hline SURF+Color & 75,21 & 67,37 & 62,65 & 59,07 & 56,12 & 53,67 & 51,39 & 49,20 & 47,03 & 44,83 & 56,65 \\
\hline CS SURF+Color & 76,94 & 68,62 & 63,87 & 60,44 & 57,60 & 55,16 & 52,99 & 50,89 & 48,71 & 46,48 & 58,17 \\
\hline
\end{tabular}

Table:2 Comparison of precision values using SURF,CS SURF, SURF + Colour and Cs SURF

\section{(6)CONCLUSION}

This paper presented a brief review about two methods for satellite image extraction using colour and texture: SURF and GLCM respectively. Image extraction using two features gives better result than extracting single feature.

\section{(7) REFERENCES}

[1] Assia Kourgli, Samia Bouteldja "High resolution satellite image indexing and retrieval using SURF features and bag of visual words" Conference Paper · March 2017

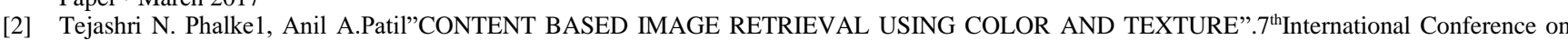
Recent Trends in Engineering, Science\&Managemet.

[3] Luca Piras, Giorgio Giacinto " Information Fusion in Content Based Image Retrieval: A Comprehensive Overview" Department of Electrical and Electronic Engineering University of Cagliari

[4] http://matlab.izmiran.ru/help/toolbox/imagrs/enhance15.html

[5] P.Mohanaiah, P.Sathyanarayana,L.GuruKumar'Image Texture Extraction Using GLCM Approach”International Journal of Scientific and Research Publication,5,May 2013.

[6] https://www.researchgate.net/figure/Main-stagesthesurf-algothim.

[7] https://en.m.wikipedia.org/wiki/speeded_up_robust_features

[8] Jacob Toft Pedersen "Study group SURF: feature detection \& description. 